\title{
Distributed Leader Selection without Direct Inter-agent Communication $\star$
}

\author{
Iman Shames * André M. H. Teixeira ${ }^{* *}$ Henrik Sandberg ${ }^{* *}$ \\ Karl H. Johansson ** \\ * The Australian National University and NICTA, Canberra, Australia \\ (e-mail: iman.shames@anu.edu.au). \\ ** The Royal Institute of Technology (KTH), Stockholm, Sweden (e-mail: \\ \{andretei,hsan,kallej\}@kth.se)
}

\begin{abstract}
This paper is an endeavour to address the problem of distributed leader selection in a formation of autonomous agents where the agents do not communicate directly via communication channels. The algorithm that the agents use to select a leader relies on the agents observing each others' behaviours. It is shown that the proposed algorithm is terminated, on average, in finite number of step and results in the selection of a leader for the formation. Moreover, It is established that the algorithm has some common elements with an algorithm widely used in data networks, i.e. Slotted Aloha. The application of the algorithm to a formation controlled by a nonlinear control law is studied and some numerical examples are presented to show the general performance of the algorithm.
\end{abstract}

Keywords: Distributed Control, Estimation Algorithms, Algorithms, Decentralized Systems, Co-operation

\section{INTRODUCTION}

Throughout history studying the behaviour of animals has inspired engineers and scientist to devise new techniques and methodologies to accomplish tasks and solve problems that were considered to be extremely challenging earlier. In recent years, a field that was heavily influenced by the knowledge obtained from observing natural creatures is the field of control and coordination of multi-agent systems. Due to the attention that this field has attracted and the knowledge that followed this spike of attention, more and more engineering problems are being addressed by the methodologies developed in this field, and it has proved to be applicable in many areas. To name a few of the application areas one can mention, sensor deployment Martınez (2009), distributed averaging and consensus Ren et al. (2007), motion coordination via flocking behaviours Jadbabaie et al. (2003); Blondel et al. (2005); Cao et al. (2008); Tanner et al. (2007), robotics formation motion control and coordination Desai et al. (2001), clock synchronization in wireless networks, see Sommer and Wattenhofer (2009).

To accomplish some of the abovementioned tasks it is required that one agent or a subset of them act as leaders of the group. By leader we mean an agent that has access to more information than the other ordinary agents. For example, an agent should act as reference clock, in clock synchronization (This leader agent "knows" the global time of the network.) or an agent's heading should be considered as the reference heading when the agents are accomplishing flocking (this agent knows what the desired direction of motion for the formation is). Such behaviours are

\footnotetext{
$\star$ This work was supported in part by the European Commission through the VIKING project, the Swedish Research Council, the Swedish Foundation for Strategic Research, the Knut and Alice Wallenberg Foundation, and NICTA which is funded by the Australian Government as represented by the Department of Broadband, Communications and the Digital Economy and the Australian Research Council through the ICT Centre of Excellence program.
}

observable in the animal kingdom as well Couzin et al. (2005), e.g. the alpha male in a pack of wolves determine where the pack should head for accomplishing foraging activities, see Mech (2000). The role and importance of leaders in the multiagent systems are well-known as well Wang and Slotine (2006), and in many scenarios the presence of a leader seems to be crucial for achieving the desired objective, Tanner et al. (2004). Since, success in accomplishing task depends on choosing a leader, one may ask how this leader is being selected in multiagent systems. There are two different ways readily available in the literature to answer this question. The first one is that the roles of the agents in the system and their relation to the other agents can be explicitly assigned to each of them, e.g. see Soukieh et al. (2009). The second way is via a distributed algorithm known as FLOODMAX, Bullo et al. (2009). While this second way is distributed, however it assumes that the agents can easily communicate with each other and pass messages to other agents in the formation. However, if one revert to animal kingdom to seek an answer to this question, he may find that the roles in groups of animals are neither assigned explicitly nor any voting-like mechanism is involved to choose a leader. The way that animals accomplish leader selection is via observing each others' behaviours, and usually the strongest one is acknowledged as the dominant member of the group (leader), King et al. (2009). This paper is an endeavour to answer the aforementioned problem in the context of multiagent formation in a way similar to that of animals.

The outline of this paper is as follows. In the next section we introduce some assumptions and state the problem that we address in this paper. In Section 3, we propose a general algorithm to address the problem posed in Section 2 and we present some analysis for the proposed algorithm. In Section 4 we address the problem of leader selection via observation for a special case of inter-agent interaction law. We show the 
applicability of the solution in Section 5 via presenting some simulation results. Concluding remarks come in the end.

\section{PROBLEM FORMULATION}

Consider a formation of $n$ interconnected nodes and let $\mathcal{G}(\mathcal{V}, \mathcal{E}, \mathcal{A})$ be the underlying graph of this formation, where $\mathcal{V}=\{i\}_{1}^{n}$ is the vertex set with $i \in \mathcal{V}$ corresponding to node $i, \mathcal{E}$ is the edge set of the graph, and $\mathcal{A} \in \mathbb{R}^{n \times n}$ is the weighted adjacency matrix with nonnegative entries. The undirected edge $\{i, j\} \in \mathcal{E}$ is incident on vertices $i$ and $j$ if nodes $i$ and $j$ share a a sensing link, in which case the corresponding entry in the adjacency matrix $\mathcal{A}_{i j}$ is positive and reflects the edge weight. The out-degree of node $i$ is $\operatorname{deg}(i)=\sum_{j \in N_{i}} \mathcal{A}_{i j}$, where $N_{i}=\{j \in \mathcal{V}:\{i, j\} \in \mathcal{E}\}$ is the neighborhood set of $i$. The degree matrix $\Delta(\mathcal{G}) \in \mathbb{R}^{n \times n}$ is a diagonal matrix defined as

$$
\Delta_{i j}= \begin{cases}\operatorname{deg}(i) & , i=j \\ 0, & i \neq j\end{cases}
$$

The weighted Laplacian of $\mathcal{G}$ is defined as $\mathcal{L}(\mathcal{G})=\Delta-\mathcal{A}$.

In this paper we consider that each agent $i$ is considered to be a single integrator and is associated with a state variable $x_{i}(t)$ at time $t$. We obtain $x(t)$ by stacking $x_{i}(t)$ for all $i \in\{1, \cdots, n\}$. Moreover for each $x_{i}(t)$ we have

$$
\dot{x}_{i}(t)=R_{i}(x(t))
$$

where $R_{i}():. \mathbb{R}^{n} \rightarrow \mathbb{R}$ is a function that relates the evolution of $x_{i}(t)$ to the other agents in the formation. Usually this relationship is limited to those $x_{j}(t)$ that $j \in N_{i}$. Hence, for all the agents' states in the formation we can consider the following general interconnection law

$$
\dot{x}(t)=R(x(t))
$$

where $R():. \mathbb{R}^{n} \rightarrow \mathbb{R}^{n}$, is the interconnection control law governing the formation. In most cases $R($.$) is directly related$ to $\mathcal{L}$, the Laplacian of the underlying graph of the formation. An example for $R($.) can be seen in the well-known consensus protocol:

$$
\dot{x}(t)=-\mathcal{L} x(t) .
$$

Definition 1 (Leader Action \& Set of Admissible Leader Actions). We say agent $i$ is performing a leader action after time $\bar{\tau}_{i}$ iff

$$
\dot{x}_{i}(t)=R_{i}(x(t))+\psi_{i}(t) \ell_{i}(t)
$$

where $R_{i}(x(t))$ is the $i$-th entry of $R(x(t))$,

$$
\ell_{i}(t)=\left\{\begin{array}{c}
f_{i}(t) \quad t \geq \bar{\tau}_{i} \\
0 \quad \text { otherwise }
\end{array}\right.
$$

where $f_{i}(t) \in \mathcal{F}$ is a real scalar function, $\left\|f_{j}(t)\right\|<M$, $\|$. denotes the Euclidian norm, and $\psi_{i}(t)$ is a Boolean variable, which is equal to 1 if agent $i$ can bid for leadership, and it is equal to 0 if it cannot. Moreover, we call $\mathcal{F}$ the set of admissible leader actions.

Definition 2 (Leader Signature). We call $f_{i}(t)$ the leader signature of agent $i$.

Assumption 1. There exists no pair $(i, j)$ with the same leader signature.

Furthermore, we have the following assumption for what each agent measures.

Assumption 2 (Agent Measurements). Each agent $i$ measures

$$
y_{i}(t)=C_{i} x(t)
$$

where $C_{i}$ is a full row rank fat matrix with $\left|N_{i}\right|$ rows and each row has all zero entries except a 1 at the $j$-th entry if $\{i, j\} \in \mathcal{E}$.
Remark 1. The definition of $\mathcal{F}$ depends on $R($.$) and all C_{i}$ and should be defined independently for different $R($.$) and$ considering all different $C_{i}$. We present an example for the conditions on $\mathcal{F}$ later.

Now consider the following system

$$
\dot{x}(t)=R(x(t))+\psi \ell(t)
$$

where $\psi=\operatorname{diag}\left(\psi_{1}, \cdots, \psi_{n}\right)$, and $\ell(t)$ is obtained by stacking all $\ell_{i}(t)$ respectively. Term a formation with a leader, $\psi$-formation. We have the following definition for this formation.

Definition 3 ( $\psi$-formation). A $\psi$-formation is a formation where all the entries of $\psi$ is zero except for exactly one equal to 1 diagonal entry. This definition is equal to the case where only one agents is able to take a leader action.

Now we propose the problem we address in this section.

Problem 1 (Distributed Leader Selection). Consider a formation of $n$ agents under the interconnection law (2) and with measurements of the form (6). How can a leader be chosen without having neither explicitly assignment of the roles to the agents nor direct communication between the agents, or equivalently how can the formation transformed into a $\psi$-formation?

\section{PROPOSED ALGORITHM}

A concept that we want to take advantage of in addressing Problem 1 is to propose an algorithm in which the agents which are able to bid for leadership are both greedy and reasonable simultaneously. We formalize these concepts in what follows.

Definition 4 (Agents Able to Bid for Leadership). An agent is able to bid for leadership if $\psi_{i}(t)=1$. Otherwise it is not able and will not bid for leadership.

Definition 5 (Greedy Agent). An agent is greedy in the context of this paper, if it wants to bid for leadership itself.

Definition 6 (Reasonable Agent). An agent is reasonable in the context of this paper, if (i) it realizes that if other agents bid for leadership prior to itself, it will not bid for leadership again, and (ii) if this agent and some other ones bid for leadership simultaneously, it will stop bidding for a bounded and random period of time and possibly bid again.

In order for the agents to act reasonably they need to be able to see if other agents are taking leadership actions. To this end we assume that each agent estimates the states of the other agents in the formation using the local measurements (6). Moreover, since each agent is assumed to be greedy, it wants to be the leader. Hence, the model that each of the agents has for the formation is a model that indicates itself as the leader, in other words, each agent $i$ considers system to be

$$
\dot{x}(t)=R(x(t))+\phi_{i}(t)
$$

where $\phi_{i}(t)=b_{i} \psi_{i}(t) \ell_{i}(t), b_{i} \in \mathbb{R}^{n}$ is a vector with all zero entries except for a 1 at the $i$-th entry. For this system, agent $i$ computes an estimate of the states, $\hat{x}^{i}(t)$ :

$$
\dot{\hat{x}}^{i}(t)=\hat{R}_{i}\left(\hat{x}^{i}(t), y_{i}(t)\right)+\phi_{i}(t) \text {. }
$$

This estimator gives rise to an error dynamics

$$
\dot{e}^{i}(t)=S_{i}\left(e^{i}(t), \phi_{i}(t), y_{i}(t)\right)
$$

where $e^{i}(t)=x(t)-\hat{x}^{i}(t)$. If the state estimators are designed in a way that the estimate values converge to the real values exponentially, $e^{i}(t)$ goes to zero exponentially as well. Now, consider the case where there are two agents $i$ and $j$, that are taking leadership actions. In this case, the estimate errors $e^{i}(t)$ and $e^{j}(t)$ do not go to zero anymore. The error dynamics at agent $i$ becomes 


$$
\dot{e}^{i}(t)=S_{i}\left(e^{i}(t), \phi_{i}(t), y_{i}(t)\right)+E^{i}(t)
$$

where $E^{i}(t)$ is a vector of zero entries except for at least one nonzero entry at the $j$-th entry $(j \neq i)$ where $E_{j}^{i}(t)=f_{j}(t)$. Hence, we have $\left\|E^{i}(t)\right\| \leq M$ where $\|$.$\| denotes the Euclidian$ norm. Of course the worst bound happens when $E^{i}(t)$ has all nonzero entries except the $i$-th one, and for this worst case, there is a time $t_{i}$, after which there exists an $\varepsilon_{i} \in \mathbb{R}_{>0}$ such that $\left\|e^{i}(t)\right\| \leq \varepsilon_{i}(n-1) M$, see Khalil and Grizzle (1996) for more detail. The agents can use the magnitude of error that they observe as an indication that there is at least one other agent in the formation bidding for leadership. We borrow a term from fault detection literature and name this error between the measured states and the estimated states, the measurement residual of the observer and we define it as

$$
r^{i}(t)=C_{i} e^{i}(t)
$$

This idea is the corner stone of the algorithm that is proposed here to answer Problem 1. However, before introducing the algorithm we need to introduce some new definitions

Definition 7 (Error Minimum Rise Time). Call the solution to

$$
\begin{array}{ll}
\max _{i} \min _{\forall E^{i}(t)} \theta_{i} & \\
\text { s.t. } & \frac{1}{\theta_{i}} \int_{0}^{\theta_{i}}\left\|r^{i}(t)\right\| d t>\delta \\
& E_{i}^{i}(t)=0 \\
& \left\|E^{i}(t)\right\| \leq M \\
& e^{i}(0)=0
\end{array}
$$

for some positive $\delta$, the error minimum rise time and denote it by $\theta^{\star}$.

Definition 8 (Error Maximum Settling Time). Call the solution to

$$
\begin{array}{ll}
\max _{i} \min _{\forall e^{i}(0)} \theta_{i} & \\
\text { s.t. } & \frac{1}{\theta_{i}} \int_{0}^{\theta_{i}}\left\|r^{i}(t)\right\| d t<\delta \\
& E^{i}(t)=0 \\
& \left\|e^{i}(0)\right\|=\varepsilon_{i}(n-1) M
\end{array}
$$

for some positive $\delta$, the error maximum settling time and denote it by $\bar{\theta}^{\star}$.

Definition 9 (Rise-Settle Time). Call $T=\left\lceil\max \left\{\bar{\theta}^{\star}, \theta^{\star}\right\}\right\rceil$ the rise-settle time.

We describe $\theta^{\star}$ and $\bar{\theta}^{\star}$ in a more intuitive way in what follows. The time it takes for all the agents in the system "sense" the effect of a perturbation in their system model, and consequently see a difference in the measured signal and the estimated signal is $\theta^{\star}$. And the time that it takes for all the observer errors to become less than a threshold in average with an "large" initial value for the error is $\bar{\theta}^{\star}$. Since, we want the agents to observe the behaviour of the system after the transient behaviours are settled we assume that measurement is carried out over a maximum time period of $T$.

Now we are ready to propose Algorithm 1 to address Problem 1 , and each agent $i$ has a copy of the algorithm. The basic idea in this algorithm is that each agent either carries out an action or makes a decision at the beginning of certain time intervals, or "time slots". These length time slots are selected in a way to allow for the agents observers to observe the states of the system after a transient time. We choose the length of the time slots to be $2 T$.

At time $t=0$, each agent $i$ chooses a random uniformly distributed integer number between 1 and $N \in \mathbb{Z}_{\geq 0}, \tilde{t}_{i}$, this number is the number of the time slot that it waits until at the beginning of which the agent starts carrying out a leader action, i.e. $\bar{\tau}_{i}=2 \tilde{t}_{i} T$, in case it had not detected any other leader action during the previous time slot. If at the beginning of the next time slot, any of the agents detect an average error in their observers during the last $T$ seconds they stop bidding for leadership from that time on. However if the error is sensed at the end of a time slot in which the agent was carrying out the leader action, this agent stops the leader action for a random period of time $\left(\bar{\tau}_{i}\right.$ is modified to reflect this idle time through choosing a new $\tilde{t}_{i}$ ), or "waits", and if till this time it does not detect any other leader action in the formation it starts acting as a leader again, otherwise it will stop any future leadership bids as before. This process continues until an agent remains as the only agent capable of bidding for leadership. We say agent $i$ is selected as a leader if its Leader $_{i}$ flag is equal to 1 .

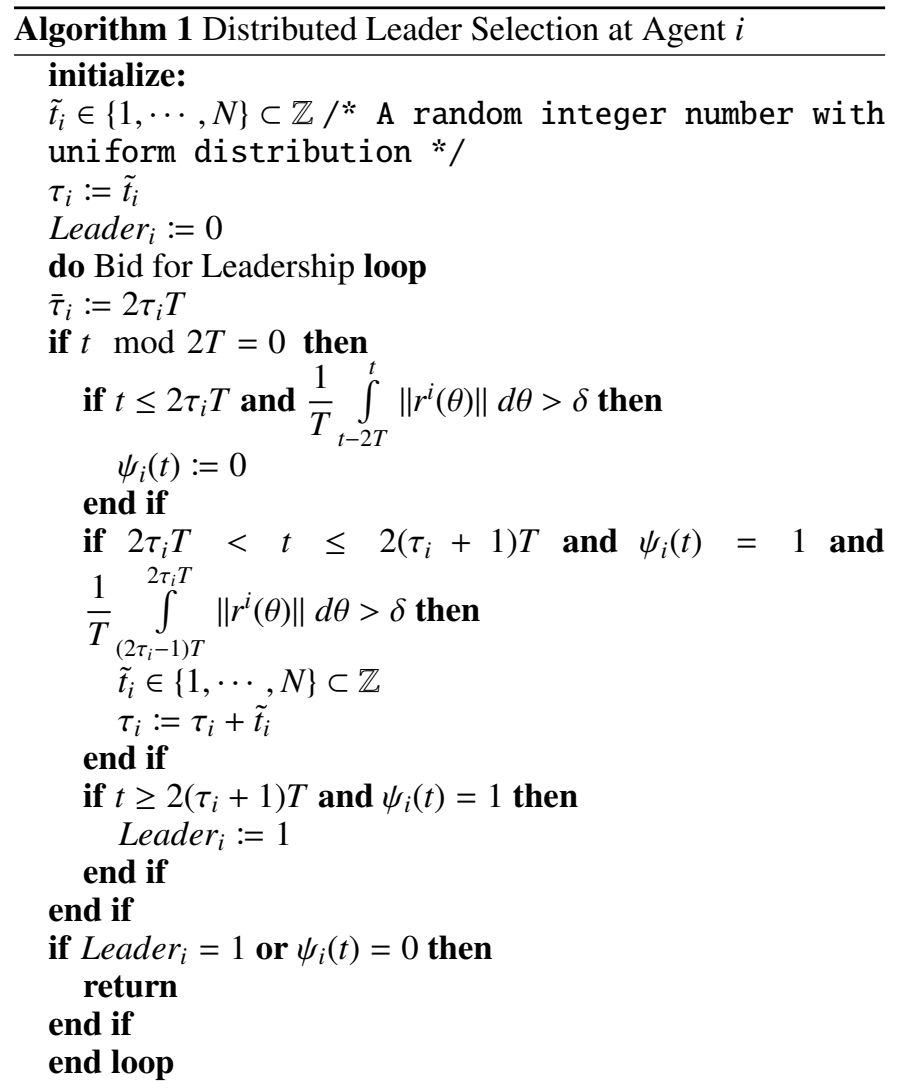

Remark 2 (Favouring the Highly Connected Agents). Intuitively it is better to have the leader connected to as many agents as possible. Hence, one can introduce different distributions for the random number generator in order to produce smaller waiting times for the agents with high connectivity. For example, $\tilde{t}_{i}$ can be chosen from the set $\left\{1, \cdots, 1+\left\lceil\frac{N}{\operatorname{deg}(i)}\right\rceil\right\}$.

\subsection{Calculating an Upper Bound for $T$}

Consider a formation of $n$ agents, one expects that the large $T$ occurs when the underlying graph of the formation is weakly connected. Among all the connected graphs, a line graph has 
the weakest connectivity. Now if we construct a line graph $\mathcal{T}$ and put agents 1 and $n$ at both ends of it. A motion generated in 1 takes the longest time to change the observer error average over time. The time that this average is larger than $\delta$ can be considered as $\theta^{\star}$. Now at the moment that it happens if the output of the agent goes to zero, the time that takes for the error average to go under $\delta$ gives us an estimate of $\bar{\theta}^{\star}$. And so we can estimate $T$ as the ceiling of the maximum of these two values.

\subsection{Comparison with Slotted ALOHA}

In this section we compare this algorithm to an algorithm used commonly in data networks, namely Slotted ALOHA. Slotted Aloha is used to provide a packet collision avoidance mechanism in data transmission, and it is simple to describe. The idea is that each station in the network sends a data packet in the beginning of prescribed time frames, called time slots, to another station in the network. If the transmitted packet collides with other data packets in the network, then the station that has sent the packet waits for a randomly generated number of time slots and retransmit again with this waiting time has elapsed Ghez et al. (1988).

One can make an analogy between the algorithm introduced here and the Slotted ALOHA. If the leader action is considered to be a data packet, taking this action is considered transmission, and the concurrent leader action by different agents is considered collision, the algorithm proposed here aim to make sure that one and only one packet is delivered without collision. However, there are some important differences between Slotted Aloha and the distributed leader selection algorithm proposed here. The most important difference is that in the leader selection algorithm if at any given time a leader is selected (the "packet" is delivered by any of the agents); the rest of the agents stop bidding for leadership (the rest would stop "transmission") indefinitely.

\subsection{Average Number of Steps Before the Termination of the Algorithm}

We consider the state in Markov chain where only one agent remains able to bid for leadership as the termination state. Thus, if the system enters this state, with probability 1 it will remain in this state. Such state is called absorbing. We define $n$ different states, and each state $i \in\{1, \cdots, n\}$ is associated with the state where $i$ agents can bid for leadership. The transition between a state $i$ to $j$ is possible with probability $p_{i, j}$ only if $j<i$. Hence, for $i<j, p_{i, j}=0$, for $j<i$ we have

$$
p_{i, j}=\left(\begin{array}{c}
i \\
j
\end{array}\right) p^{j}(1-p)^{i-j},
$$

where $p=1 / N$, while for $i=j$ we have

$$
p_{i, i}=1-\sum_{j=1}^{i-1}\left(\begin{array}{l}
i \\
j
\end{array}\right) p^{j}(1-p)^{i-j} .
$$

Defining these probabilities and states enable us to put the states that the system transitions between as a Markov chain. This chain is depicted in Fig. 1.

Define matrix $P$, with entries $p_{i j}$, as the state transition matrix of an absorbing Markov chain with state transition probability of $p_{i, j}$. Putting the transition matrix into canonical form we have

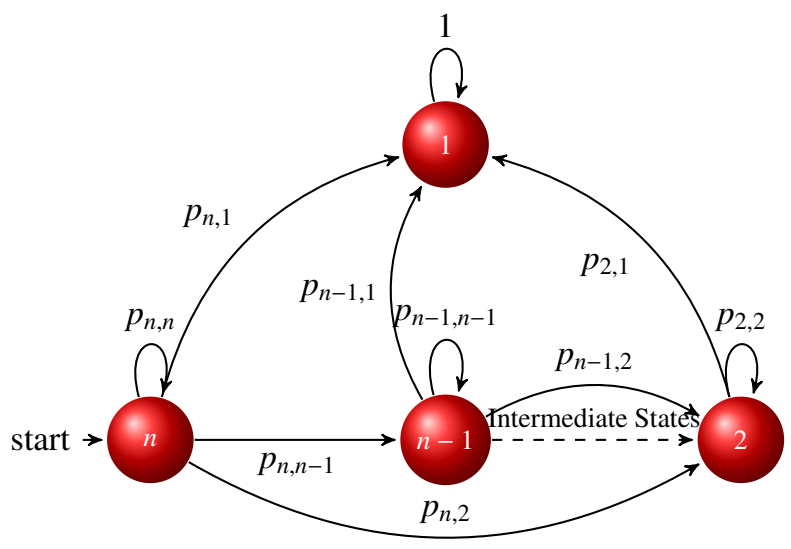

Fig. 1. The Markov chain corresponding to the states that the system goes through under Distributed Leader Selection Algorithm.

$$
\begin{aligned}
P_{c} & =\left[\begin{array}{ccc|c}
1-2 p(1-p) & 0 & \ldots & 2 p(1-p) \\
\vdots & \vdots & \ddots & \vdots \\
\left(\begin{array}{c}
n \\
2
\end{array}\right) p^{2}(1-p)^{n-2} & \ldots & \ldots & n p(1-p)^{n-1} \\
\hline 0 & \ldots & 1
\end{array}\right] \\
& =\left[\begin{array}{c|c}
Q & W \\
\hline 0_{1 \times n-1} & 1
\end{array}\right]
\end{aligned}
$$

where $P_{c}$ is canonical form of $P$. From Grinstead and Snell (1997) we have the following result.

Proposition 1 (Time to Absorption). Let $\bar{t}_{i}$ be the expected number of steps before the chain is absorbed, given that the chain starts in state $i$, and let $\bar{t}$ be the column vector whose ith entry is $\bar{t}_{i}$. Then

$$
\bar{t}=(I-Q)^{-1} \mathbf{1},
$$

where $I$ is the identity matrix and $\mathbf{1}$ is a column vector of appropriate dimension with all entries of 1 .

Algorithm 1 is terminated (on average) in the same steps as the abovementioned Markov chain is absorbed. The entry $\bar{t}_{i}$ is the average value for steps until termination when the chain is initialized at state $i$. Hence on average Algorithm 1 is terminated in finite steps, and as the number of steps goes to infinity the termination probability of the algorithm goes to 1 as well.

For example the average number of steps for Algorithm 1 for a formation of $n=10$ agents, with $N=10$ to have a leader from the initial 10 potential leaders is 25 steps.

Remark 3. One might implement the algorithm letting only a subset of the agents bid for leadership, rather than all of them. A possible scenario that this may be desirable is; to replace a dysfunctional leader and letting only the neighbours of the previous leader to bid for leadership. One can choose the number of the agents in this subset based on the entries of $\bar{t}$.

\section{LEADER SELECTION IN FORMATIONS WITH NONLINEAR CONSENSUS LAW}

Consider a formation of $n$ single integrators under the following consensus law which is based on the tracking consensus law proposed in Cao and Ren (2010)

$$
\dot{x}(t)=-\mathcal{L} x(t)-\beta \operatorname{sgn}(\mathcal{L} x(t))
$$


where $\beta \in \mathbb{R}_{>0}$. To address Problem 1 , we first need to construct an observer for this system.

For each agent $i$ we have the following system model

$$
\begin{aligned}
\dot{x}(t) & =-\mathcal{L} x(t)-\beta \operatorname{sgn}(\mathcal{L} x)+\phi_{i}(t) \\
y_{i}(t) & =C_{i} x(t) .
\end{aligned}
$$

where $\phi_{i}(t)=b_{i} \psi_{i}(t) \ell_{i}(t)$, and $\operatorname{sgn}($.$) operator is considered to$ be a vector version of ordinary scalar $\operatorname{sgn}($.$) operator. We can$ rewrite the system as

$$
\begin{aligned}
\dot{x}(t) & =A x(t)+G \rho(A x(t))+\phi_{i}(t) \\
y_{i}(t) & =C_{i} x(t),
\end{aligned}
$$

where $A=-\mathcal{L}, G=\beta$, and $\rho()=.\operatorname{sgn}($.$) .$

From Arcak and Kokotovic (2001); Fan and Arcak (2003) we know that we can construct an observer for this system where $\rho($.) should satisfy the following assumption.

Assumption 3. The function $\rho(v)$ satisfies the monotonicity property $\forall v \in \mathbb{R}^{n}$ iff

$$
\frac{\partial \rho}{\partial v}+\left(\frac{\partial \rho}{\partial v}\right)^{\top} \geq 0
$$

The observer is given by

$$
\begin{aligned}
\hat{x}^{i}(t) & =A \hat{x}^{i}(t)+L_{i}\left(C_{i} \hat{x}^{i}(t)-y_{i}(t)\right) \\
& +\beta \rho\left(A \hat{x}^{i}(t)+K_{i}\left(C_{i} \hat{x}^{i}(t)-y_{i}(t)\right)\right)+\phi_{i}(t)
\end{aligned}
$$

Moreover, we know that the error associated with this observer goes to zero if the following linear matrix inequality (LMI) has a solution for $P_{i}>0, \kappa_{i}>0, K_{i}$, and $L_{i}$.

$$
\left[\begin{array}{cc}
\left(A+L_{i} C_{i}\right)^{\top} P_{i}+P_{i}\left(A+L_{i} C_{i}\right)+\kappa_{i} I & \beta P_{i}+\left(A+K_{i} C_{i}\right)^{\top} \\
\beta P_{i}+\left(A+K_{i} C_{i}\right) & 0
\end{array}\right] \leq 0
$$

For the error dynamics we have

$$
\begin{aligned}
\dot{e}^{i}(t) & =\left(A+L_{i} C_{i}\right) e^{i}(t) \\
& +\beta\left(\operatorname{sgn}(A x(t))-\operatorname{sgn}\left(A \hat{x}^{i}(t)+K_{i}\left(C_{i} \hat{x}^{i}(t)-y_{i}(t)\right)\right)\right)
\end{aligned}
$$

Consequently for the residual of the observer at agent $i$ we have

$$
\begin{aligned}
\dot{r}^{i}(t) & =C_{i} L_{i} r^{i}(t)+C_{i} A e^{i}(t) \\
& +\beta C_{i}\left(\operatorname{sgn}(A x(t))-\operatorname{sgn}\left(A \hat{x}^{i}(t)+K_{i}\left(C_{i} \hat{x}^{i}(t)-y_{i}(t)\right)\right)\right)
\end{aligned}
$$

Under (19) for the set of admissible leader action, $\mathcal{F}$ we have:

Definition 10 (Set of Admissible Leader Actions for (19)). The set $\mathcal{F}$ is defined as the set of all scalar functions such that for any $f_{j}(t) \in \mathcal{F}$, all $C_{i}$, and for almost all $t$ and $\forall i, j \in\{1, \cdots, n\}$ there exists a positive number $c_{1}$ such that

$$
0<\left\|\mu_{i j}(t)\right\|^{2} \leq c_{1}
$$

where

$$
\begin{aligned}
\mu_{i j}(t) & =C_{i} A e^{i}(t) \\
& +\beta C_{i}\left(\operatorname{sgn}(A x(t))-\operatorname{sgn}\left(A \hat{x}^{i}(t)+K_{i}\left(C_{i} \hat{x}^{i}(t)-y_{i}(t)\right)\right)\right.
\end{aligned}
$$

Before showing that such observer exists for the system described by (21) we present the following two lemmas whose proofs are omitted for brevity.

Lemma 1 (Rank of Laplacian Principal Submatrices). If an undirected graph $\mathcal{G}$ is connected, then any principal submatrix of its Laplacian matrix $\mathcal{L}$, induced by a strict subset of nodes $\overline{\mathcal{V}} \subset \mathcal{V}$, is invertible.

Lemma 2. Consider $\mathcal{L} \in \mathbb{R}^{n \times n}$ to be a Laplacian matrix associated with a connected graph. Further define $X \geq 0$ to be a matrix of the same dimension as $\mathcal{L}$ where all the entries are 0 except for possibly its diagonal entries. Moreover it is assumed that at least a diagonal entry of $X$ is equal to 1 . Then for any $\lambda>0, \mathcal{L}_{\lambda}=\mathcal{L}+\lambda X$ is positive definite.

Lemma 3 (LMI Feasibility). There exist $P_{i}=P_{i}^{\top}>0, \kappa_{i}>0$, $K_{i}$ and $L_{i}$ of appropriate dimensions such that (24) holds.

Proof. This problems is equivalent to

$$
\begin{aligned}
\beta P_{i}+\left(A+K_{i} C_{i}\right) & =0 \\
\left(A+L_{i} C_{i}\right)^{\top} P_{i}+P_{i}\left(A_{i}+L_{i} C_{i}\right) & <0
\end{aligned}
$$

We claim that $L_{i}=K_{i}=\lambda C_{i}^{\top}$ and $P_{i}=-\left(A+\lambda C_{i}^{\top} C_{i}\right) / \beta$ satisfy these equations, where $\lambda \in \mathbb{R}_{<0}$. To check this claim, first notice that $A=-\mathcal{L}$, hence $P_{i}=\left(\mathcal{L}-\lambda C_{i}^{\top} C_{i}\right) / \beta$. From Lemma 2 we know that then $P_{i}$ is strictly positive. Furthermore for the second inequality we have

$$
-P_{i}^{\top} P_{i}+P_{i} P_{i}^{\top}=-2\left(P_{i}\right)^{2}<0 .
$$

This shows that the LMI is feasible.

The next theorem establishes that the observer error described by (25) goes to zero exponentially fast for system (21), if no other agents take a leader action.

Theorem 1 (The Exponential Stability of the Nonlinear Observer). The observer error, $e^{i}(t)$ described by (25) goes to zero exponentially fast for system (21).

Proof. We know from Fan and Arcak (2003) that if Assumption 3 is satisfied and LMI (24) is feasible then the error dynamics (25) is exponentially stable. To check the former note that $\operatorname{sgn}($. is non-decreasing and hence the Assumption is satisfied and Lemma 3 shows that the latter holds as well.

Now since we can construct observers at each agent with the local information available to that agent to monitor the states of the network, we can detect if the observer output error does not go to zero as well, and in average being larger than a threshold $\delta \in \mathbb{R}_{>0}$. Thus, we can apply Algorithm 1 to select a leader in the formation under control law (19). In the next section we present some simulation results showing the performance of of Algorithm 1 for selecting a leader in the formations controlled by (19).

\section{SIMULATIONS}

In this section we consider a leader selection scenario for the nonlinear consensus protocol introduced by (19).

In the first scenario consider a formation of $n=10$ agents with leader signature $f_{i}(t)=40 i \sin (2 i t), L_{i}=K_{i}=-20 C_{i}^{\top} C_{i}$ ( $i \in\{1, \cdots, n\}$ ), and $N=5$. Moreover, $T$ is considered to be $2 \mathrm{sec}$ and hence the time slot is $4 \mathrm{sec}$. The process of leader selection is depicted in Fig. 2.

In the second scenario we aim to present the motion of the agents when they run the leader selection algorithm. We consider again a formation of $n=10$ agents with leader signature $f_{i}(t)=40 i \sin (2 i t), L_{i}=K_{i}=-20 C_{i}^{\top} C_{i}(i \in\{1, \cdots, n\}), N=5$, and $T=2 \mathrm{sec}$. The formation at time $t=0$ is depicted in Fig 3 (a) and the motion of the agents $x$ coordinates is presented in Fig 3(b). 

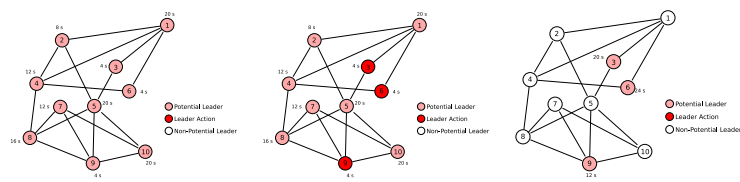

(a) Agents at time $t=$ (b) Agents at time $t=$ (c) Agents at time $t=$ 0



(d) Agents at time $t=($

12

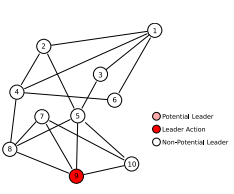

Fig. 2. The underlying graph of the formation. The numbers on the circles denote the agents labels, and the smaller ones outside the circles are the time they will take their corresponding leader action.



(a) Formation at time $t=0$

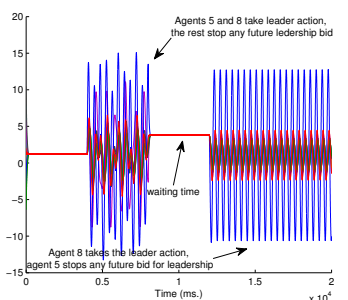

(b) The motion of the agent throughout the leader selection process
Fig. 3. The motion of the agents throughout the leader selection process in the second scenario.

\section{CONCLUDING REMARKS AND FUTURE DIRECTIONS}

In this paper we proposed an algorithm for leader selection in multi-agent formations. The algorithm requires only local measurements and a knowledge of the structure of the underlying graph of the formation at each agent. The algorithm relies on existence of state observers at each agent that can estimate the states of other agents in the formation, and on a suitable set of admissible leader actions. We have shown that such observers exist for a nonlinear interaction law. We further demonstrated the applicability of the algorithm via simulation results. A future research problem is to consider directed fixed and/or switching topologies.

\section{ACKNOWLEDGEMENTS}

The authors would specially thank Prof. Wei Ren for his valuable comments on the role of leaders in the motion coordination of a formation of agents.

\section{REFERENCES}

Arcak, M. and Kokotovic, P. (2001). Nonlinear observers: a circle criterion design and robustness analysis. Automatica, 37(12), 1923 - 1930. doi:DOI: 10.1016/S00051098(01)00160-1.

Blondel, V.D., Hendrickx, J.M., Olshevsky, A., and Tsitsiklis, J.N. (2005). Convergence in multiagent coordination, consensus, and flocking. In 44th IEEE Conference on Decision and Control, 2005 and 2005 European Control Conference. CDC-ECC'05, 2996-3000.

Bullo, F., Corts, J., and Martnez, S. (2009). Distributed Control of Robotic Networks, Series in Applied Mathematics. Princeton.

Cao, M., Morse, A.S., and Anderson, B.D.O. (2008). Reaching a consensus in a dynamically changing environment: Convergence rates, measurement delays, and asynchronous events. SIAM J. Control Optim., 47(2), 601-623.

Cao, Y. and Ren, W. (2010). Distributed coordinated tracking via a variable structure approach -part $\mathrm{i}$ : Consensus tracking.

Couzin, I.D., Krause, J., Franks, N.R., and Levin, S.A. (2005). Effective leadership and decision-making in animal groups on the move. Nature, 433(7025), 513-516.

Desai, J.P., Ostrowski, J.P., and Kumar, V. (2001). Modeling and control of formations of nonholonomic mobile robots. IEEE transactions on Robotics and Automation, 17(6), 905908.

Fan, X. and Arcak, M. (2003). Observer design for systems with multivariable monotone nonlinearities. Systems and Control Letters, 50(4), 319.

Ghez, S., Verdu, S., and Schwartz, S.C. (1988). Stability properties of slotted Aloha with multipacket reception capability. IEEE Transactions on Automatic Control, 33(7), 640-649.

Grinstead, C. and Snell, J. (1997). Introduction to Probability, chapter 11. Markov Chains. American Mathematical Society, Pacific Grove, California.

Jadbabaie, A., Lin, J., and Morse, A.S. (2003). Coordination of groups of mobile autonomous agents using nearest neighbor rules. IEEE Transactions on Automatic Control, 48(6), 9881001 .

Khalil, H.K. and Grizzle, J.W. (1996). Nonlinear systems. Prentice hall Englewood Cliffs, NJ.

King, A.J., Johnson, D.D., and Vugt, M.V. (2009). The origins and evolution of leadership. Current Biology, 19(19), R911 - R916. doi:DOI: 10.1016/j.cub.2009.07.027.

Martınez, S. (2009). Distributed interpolation schemes for field estimation by mobile sensor networks. IEEE Transactions on Control Systems Technology.

Mech, L.D. (2000). Leadership in wolf, Canis lupus, packs. Canadian Field-Naturalist, 114(2), 259-263.

Ren, W., Beard, R.W., and Atkins, E.M. (2007). Information consensus in multivehicle cooperative control. IEEE Control Systems Magazine, 27(2), 71-82.

Sommer, P. and Wattenhofer, R. (2009). Gradient clock synchronization in wireless sensor networks. In Proceedings of the 2009 International Conference on Information Processing in Sensor Networks, 37-48.

Soukieh, R., Shames, I., and Fidan, B. (2009). Obstacle avoidance of robotic formations based on fluid mechanical modeling. In Proc. European Control Conference, 32633268. Budapest, Hungary.

Tanner, H.G., Pappas, G.J., and Kumar, V. (2004). Leaderto-formation stability. IEEE Transactions on Robotics and Automation, 20(3), 443-455.

Tanner, H., Jadbabaie, A., and Pappas, G. (2007). Flocking in fixed and switching networks. IEEE Transactions on Automatic Control, 52(5), 863-868.

Wang, W. and Slotine, J.J.E. (2006). A theoretical study of different leader roles in networks. IEEE Transactions on Automatic Control, 51(7), 1156-1161. 